5 Smith D, Spanel P. Selected ion flow tube mass spectrometry (SIFT-MS) for on-line trace gas analysis. Mass Spectrom Rev 2005; 24: 661-700.

6 Lee TW, Brownlee KG, Conway SP, et al. Evaluation of a new definition for chronic Pseudomonas aeruginosa infection in cystic fibrosis patients. J Cyst Fibros 2003; 2: 29-34.

7 Kumar S, Huang J, Abbassi-Ghadi N, et al. Selected ion flow tube mass spectrometry analysis of exhaled breath for volatile organic compound profiling of esophago-gastric cancer. Anal Chem 2013; 85: 6121-6128.

8 Sinha A, Krishnan V, Sethi T, et al. Metabolomic signatures in nuclear magnetic resonance spectra of exhaled breath condensate identify asthma. Eur Respir J 2012; 39: 500-502.

9 Montuschi P, Paris D, Montella S, et al. Nuclear magnetic resonance-based metabolomics discriminates primary ciliary dyskinesia from cystic fibrosis. Am J Respir Crit Care Med 2014; 190: 229-233.

10 Montuschi P, Paris D, Melck D, et al. NMR spectroscopy metabolomic profiling of exhaled breath condensate in patients with stable and unstable cystic fibrosis. Thorax 2012; 67: 222-228.

11 de Carvalho Costa Cardinali L, Rocha GA, Rocha AM, et al. Evaluation of $\left[{ }^{13} \mathrm{C}\right]$ urea breath test and Helicobacter pylori stool antigen test for diagnosis of $\mathrm{H}$. pylori infection in children from a developing country. J Clin Microbiol 2003; 41: 3334-3335.

12 Prince BJ, Milligan DB, McEwan MJ. Application of selected ion flow tube mass spectrometry to real-time atmospheric monitoring. Rapid Commun Mass Spectrom 2010; 24: 1763-1769.

13 Montuschi P, Mores N, Trove A, et al. The electronic nose in respiratory medicine. Respiration 2013; 85: 72-84.

14 Goeminne PC, Vandendriessche T, Van Eldere J, et al. Detection of Pseudomonas aeruginosa in sputum headspace through volatile organic compound analysis. Respir Res 2012; 13: 87.

15 Ryall B, Davies JC, Wilson R, et al. Pseudomonas aeruginosa, cyanide accumulation and lung function in CF and non-CF bronchiectasis patients. Eur Respir J 2008; 32: 740-747.

16 Smith D, Spanel P, Gilchrist FJ, et al. Hydrogen cyanide, a volatile biomarker of Pseudomonas aeruginosa infection. J Breath Res 2013; 7: 044001.

17 Dummer J, Storer M, Sturney S, et al. Quantification of hydrogen cyanide (HCN) in breath using selected ion flow tube mass spectrometry - HCN is not a biomarker of Pseudomonas in chronic suppurative lung disease. $J$ Breath Res 2013; 7: 017105.

18 Chen W, Metsala M, Vaittinen O, et al. Hydrogen cyanide in the headspace of oral fluid and in mouth-exhaled breath. J Breath Res 2014; 8: 027108 .

19 Robertson JM, Friedman EM, Rubin BK. Nasal and sinus disease in cystic fibrosis. Paediatr Respir Rev 2008; 9 : 213-219.

\title{
Monoallelic germline ATM mutation and organising pneumonia induced by radiation therapy to the breast
}

\author{
To the Editor:
}

Organising pneumonia is defined by the presence of intra-alveolar buds consisting of inflammatory cells, fibroblasts and myofibroblasts, and loose connective tissue, often associated with bronchiolitis obliterans. Organising pneumonia may be cryptogenic (idiopathic) or associated with a variety of causes including, especially, infection, adverse reaction to drugs, connective tissue diseases [1] and radiation therapy to the breast $[2,3]$.

Ataxia telangiectasia is an autosomal recessive disorder resulting from mutations in the ATM (ataxia telangiectasia mutated) gene located in 11q22.3, with a prevalence of about one in 100000 children. ATM encodes a 350-kDa protein kinase (3056 aminoacyl residues) with catalytic functions similar to phosphatidylinositol 3'-kinases. After DNA damage, especially DNA double-strand breaks, ATM phosphorylates a thousand targets, activating cell cycle checkpoints, DNA repair, and metabolic and senescence pathways. Ataxia telangiectasia is characterised by neurological manifestations, an increased risk of infections, and cancers, especially lymphomas and leukaemias. Ataxia telangiectasia children affected with cancer present with a severe radiosensitivity if radiation is not reduced. Women carrying a monoallelic ATM mutation, for example, the relatives of ataxia telangiectasia-affected children, are at increased risk of breast cancer [4]. The relative risk of breast cancer at age 50 years in ATM carriers has been estimated to be 4.94 (95\% CI 1.90-12.9) among relatives of ataxia telangiectasia patients [5].

A 68-year-old woman underwent tumourectomy for invasive ductal carcinoma of the left breast followed by radiation therapy, completed in September 2008. There were no acute side-effects. The patient did not receive chemotherapy. 2 months later, she developed bilateral pulmonary alveolar opacities on imaging, 
typical of radiation-induced organising pneumonia (RIOP). She declined bronchoalveolar lavage. She received $40 \mathrm{mg}$ prednisone per day, which was progressively decreased, with regression of the pulmonary opacities. However, relapse occurred in March 2009 while she was taking $5 \mathrm{mg}$ prednisone per day. Prednisone was re-escalated to $30 \mathrm{mg}$ per day, with clearing of the pulmonary opacities. Prednisone was then progressively decreased and eventually discontinued in September 2009.

The patient continued to do well and chest high-resolution computed tomography (HRCT) in February 2012 was normal. However, in September 2012, she presented with dyspnoea on exertion, dry cough and asthenia. HRCT showed reappearance of bilateral alveolar opacities (figure 1). Corticosteroid treatment was reinitiated at a dose of $60 \mathrm{mg}$ per day and then progressively decreased. A daily dose of $30 \mathrm{mg}$ prednisone was required to obtain clearing of alveolar opacities and the dose was again progressively decreased.

Another relapse occurred in May 2013 while under $15 \mathrm{mg}$ prednisone per day. After a transient increase of corticosteroid dose to $20 \mathrm{mg}$ per day, treatment was progressively decreased to $10 \mathrm{mg}$ per day, which was maintained over the long term. In April 2014, the chest radiograph was normal. It was then decided to limit further chest imaging because of the presumed risk of radiation-induced cancer.

The patient had two daughters and one son. Her older daughter was healthy. Her younger daughter and her son were affected with ataxia telangiectasia. Her daughter presented with a severe cerebellar syndrome and no clinical immune deficiency. Lymphocyte counts, and IgG2, IgG4 and IgA serum levels were normal. Lymphocyte karyotype was typical of ataxia telangiectasia with $37 \%$ of metaphases presenting with rearrangements of chromosomes 7 and 14. The serum level of $\alpha$-fetoprotein was very elevated (40 times higher than upper limit of normal). At age 22 years, she developed thyroid carcinoma. She died at the age of 30 years from inhalation pneumonitis and Pseudomonas septicaemia. Her son had cerebellar syndrome, developed T-cell prolymphocytic leukaemia at age 29 years and died a few months later. The diagnosis of ataxia telangiectasia in the daughter and son was based on cerebellar syndrome, biological immune deficiency, increased $\alpha$-fetoprotein serum level and lymphocyte chromosomal rearrangements of the immunoglobulin superfamily genes. It was confirmed by molecular analysis of the ATM gene.

Sequencing of the ATM gene in the patient's ataxia telangiectasia-affected daughter disclosed two inactivating mutations, c.2734C>T;p.Gln912* and c.8494C >T;p.Arg2832Cys [6]. The patient carried the c.2734C>T;p.Gln912* mutation. The father of the affected children carried the c.8494C>T;p.Arg2832Cys mutation, thus confirming that the two mutations were in two different alleles and that the affected daughter was a compound heterozygote. No ATM test has been performed in the son who died or in the unaffected daughter, who declined the test.

RIOP is an archetypal model of organising pneumonia, quite similar to cryptogenic organising pneumonia and very different from acute radiation pneumonitis following cancer radiotherapy, which develops during or early after completion of radiation therapy, with lesions limited to the irradiated area, eventually resulting in persisting fibrosis. In contrast, RIOP has been defined as occurring within 12 months after radiation therapy, general and/or respiratory symptoms lasting for $\geqslant 2$ weeks, radiographic lung infiltrates outside the radiation field (abscopal or distant bystander effect) and no evidence of another specific cause [3]. The prevalence of RIOP ranges from $0.8 \%$ to $2.9 \%$ of patients treated with radiation therapy to the breast $(1.68 \%$ in an extensive
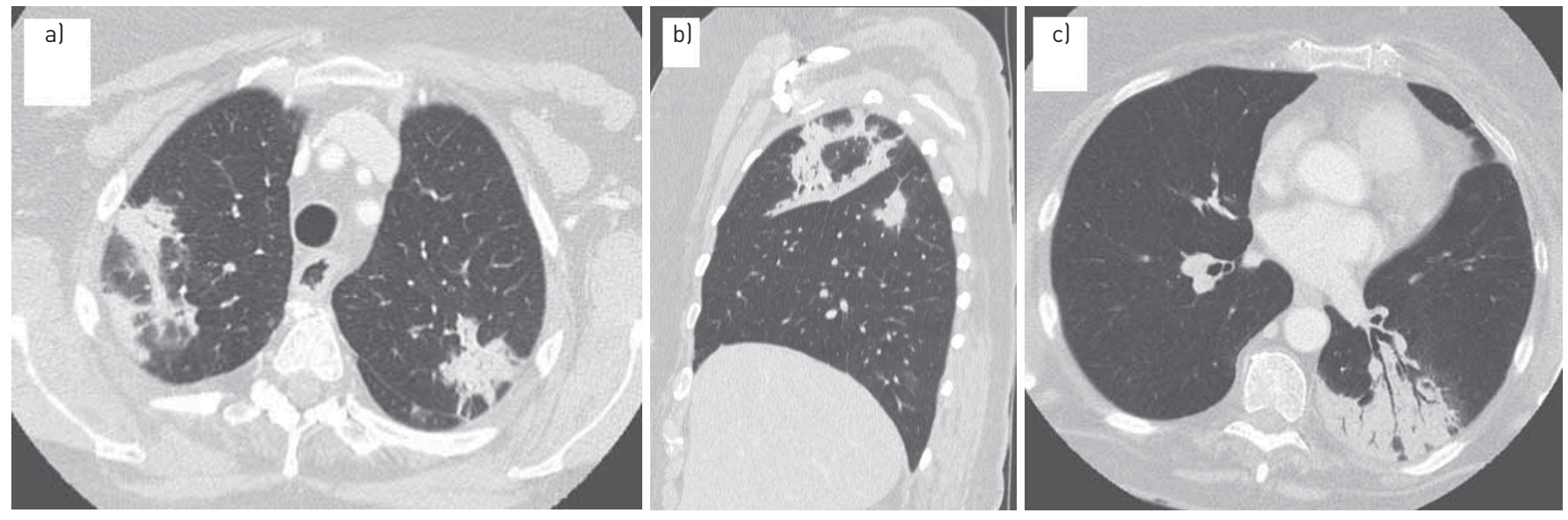

FIGURE 1 Chest computed tomography images demonstrating consolidation typical of organising pneumonia. a) Patchy bilateral opacities in the upper lobes; b) sagittal reformatted image showing ring-shaped airspace consolidation in the left upper lobe; cl consolidation with air bronchogram in the left lower lobe. 
literature review) [7]. Age $\geqslant 50$ years, concurrent anti-oestrogen therapy [8] and possibly tobacco smoking [7], but not the type of breast surgery (radical or limited), may be associated with an increased risk of RIOP. Patients with RIOP frequently present with fever, cough and dyspnoea, although some may be asymptomatic. Alveolar opacities (often with air bronchogram and migratory) are found on chest imaging [3], with frequent involvement contralateral to the irradiated breast. Most patients receive corticosteroids, with dramatic effects on clinical features and imaging abnormalities. However, multiple relapses are common, usually while decreasing the doses of corticosteroids or soon after these are discontinued [1].

Pulmonary manifestations of ataxia telangiectasia $[9,10]$ include immune dysfunction, leading to recurrent infections of the upper and lower respiratory tract, aspiration as a consequence of dysfunctional swallowing due to neurological deficit, inefficient cough and interstitial lung disease. Interstitial lung disease is present in about $25 \%$ of patients with ataxia telangiectasia, with a mean age of onset of 17.5 years. It is characterised by chronic inflammation and lung fibrosis, with a lymphocytic pattern on bronchoalveolar lavage. Treatment with systemic corticosteroids has been associated with clinical and radiographic improvement [11]. No characteristic organising pneumonia has hitherto been reported in ataxia telangiectasia patients.

Patients with ataxia telangiectasia are unusually sensitive to therapeutic doses of ionising radiation (radiosensitivity) and thus, the use of X-rays and HRCT should be limited to the lowest possible dose [9]. The minus alleles of the $A T M-111 \mathrm{G}>\mathrm{A}$ and $126713 \mathrm{G}>\mathrm{A}$ polymorphisms have been associated with an increased risk of organising pneumonia in patients who have undergone radiotherapy for lung cancer, with hazard ratios of 2.49 (95\% CI 1.07-5.8) and 2.47 (95\% CI 1.16-5.28), respectively [12]. In a 30-year-old female treated with total body irradiation and allogenic stem-cell transplantation for acute lymphoblastic leukaemia who has developed erythema, skin bullae and erosions, gastric erythema, and retinopathy because of the exceptional degree of radiation-induced toxicity, sequencing of the ATM gene showed a monoallelic nonsense mutation, c.4396C>T;Arg1466* [13].

Our patient presented a similar nonsense mutation leading to a putative truncated protein or even the absence of protein in case of the instability of the truncated mutation induced product. Although a causal relationship cannot be demonstrated, we hypothesise that it may have predisposed to RIOP. It should be noticed that the frequency of ATM mutation carriers among women affected with breast cancer has been estimated to be $2.04 \%$ [14], consistent with the frequency of RIOP in women irradiated for breast cancer. It remains to be explored whether ATM gene mutations may be found in other patients with RIOP.

RIOP has hitherto not been reported in ataxia telangiectasia patients or in individuals with monoallelic ATM gene mutations. We consider that RIOP in our patient may have resulted from the radiosensitivity associated with the ATM gene monoallelic mutation.

0 @ERSpublications

Radiation-induced organised pneumonia may be associated with monoallelic ATM gene mutation http://ow.ly/WIpFe

Jean-François Cordier ${ }^{1,2}$, Vincent Cottin ${ }^{1,2}$, Romain Lazor ${ }^{1,3}$ and Dominique Stoppa-Lyonnet ${ }^{4}$

${ }^{1}$ Dept of Respiratory Medicine, Groupement Hospitalier Est, Lyon, France. ${ }^{2}$ Claude Bernard University, Lyon, France. ${ }^{3}$ Centre Hospitalier Universitaire Vaudois, Lausanne, Switzerland. ${ }^{4}$ Dept of Genetics, Institut Curie, Paris Descartes University, Paris, France.

Correspondence: Jean-François Cordier, Hopital L. Pradel, Batiment A4 Pneumologie, Service de Pneumologie, 59 Boulevard Pinel, F-69677, Lyon, France. E-mail: jean-francois.cordier@chu-lyon.fr

Received: Nov 062015 | Accepted after revision: Dec 152015 | First published online: Feb 042016

Conflict of interest: None declared.

\section{References}

1 Cordier JF. Cryptogenic organising pneumonia. Eur Respir J 2006; 28: 422-446.

2 Bayle JY, Nesme P, Bejui-Thivolet F, et al. Migratory organizing pneumonitis "primed" by radiation therapy. Eur Respir J 1995; 8: 322-326.

3 Crestani B, Valeyre D, Roden S, et al. Bronchiolitis obliterans organizing pneumonia syndrome primed by radiation therapy to the breast. The Groupe d'Etudes et de Recherche sur les Maladies Orphelines Pulmonaires (GERM “O” P). Am J Respir Crit Care Med 1998; 158: 1929-1935.

4 Geoffroy-Perez B, Janin N, Ossian K, et al. Variation in breast cancer risk of heterozygotes for ataxia-telangiectasia according to environmental factors. Int J Cancer 2002; 99: 619-623.

5 Thompson D, Duedal S, Kirner J, et al. Cancer risks and mortality in heterozygous ATM mutation carriers. J Natl Cancer Inst 2005; 97: 813-822.

6 Mitui M, Nahas SA, Du LT, et al. Functional and computational assessment of missense variants in the ataxia-telangiectasia mutated (ATM) gene: mutations with increased cancer risk. Hum Mutat 2009; 30: 12-21. 
7 Epler GR, Kelly EM. Systematic review of postradiotherapy bronchiolitis obliterans organizing pneumonia in women with breast cancer. Oncologist 2014; 19: 1216-1226.

8 Katayama N, Sato S, Katsui K, et al. Analysis of factors associated with radiation-induced bronchiolitis obliterans organizing pneumonia syndrome after breast-conserving therapy. Int J Radiat Oncol Biol Phys 2009; 73: 1049-1054.

9 Bhatt JM, Bush A, van Gerven M, et al. A statement on the multidisciplinary respiratory management of ataxia telangiectasia. Eur Respir Rev 2015; 24: 565-581.

10 Bhatt JM, Bush A, van Gerven M, et al. Ataxia telangiectasia: why should the ERS care? Eur Respir J 2015; 46: 1557-1560.

11 Schroeder SA, Swift M, Sandoval C, et al. Interstitial lung disease in patients with ataxia-telangiectasia. Pediatr Pulmonol 2005; 39: 537-543.

12 Zhang L, Yang M, Bi N, et al. ATM polymorphisms are associated with risk of radiation-induced pneumonitis. Int J Radiat Oncol Biol Phys 2010; 77: 1360-1368.

13 Ostendorf BN, Terwey TH, Hemmati PG, et al. Severe radiotoxicity in an allogeneic transplant recipient with a heterozygous ATM mutation. Eur J Haematol 2015; 95: 90-92.

14 Renwick A, Thompson D, Seal S, et al. ATM mutations that cause ataxia-telangiectasia are breast cancer susceptibility alleles. Nat Genet 2006; 38: 873-875.

\title{
Regimens for nontuberculous mycobacterial lung disease lack early bactericidal activity
}

\author{
To the Editor:
}

Over recent decades, nontuberculous mycobacteria (NTM) have been increasingly recognised as causative agents of pulmonary infections in humans [1]. Mycobacterium avium complex (MAC) bacteria are the most common NTM species causing pulmonary disease in humans [1]. Pulmonary disease caused by NTM (NTM-PD) usually presents as either nodular-bronchiectatic or fibrocavitary disease. NTM-PD requires complex multidrug antibiotic treatment to be continued for 12 months after culture conversion, typically for 18 months in total [1]. Despite this intensive treatment, good clinical and microbiological outcomes can only be attained in $60 \%$ of patients with MAC pulmonary disease [2]. Outcomes in nodular-bronchiectatic MAC pulmonary disease are better, with prolonged culture conversion attainable in up to $85 \%$ of patients [3].

The long treatment regimen, and the bias introduced by morbidity and mortality related to the patients' comorbid conditions and comedications, complicate the use of long-term outcome measures in clinical trials. Hence, there is a need for a robust marker that can be measured early in treatment and that predicts the long-term outcome of treatment. One such marker could be the early bactericidal activity (EBA). EBA studies are based on the principle that the effect of treatment in the first days or weeks on the bacillary load in clinical samples predicts the long-term result of treatment. The treatment of tuberculosis has been optimised using this principle [4].

If they prove to be good predictors of long-term outcomes, EBA studies could greatly decrease the time required to assess the effect of treatment. In this pilot study, we aimed to measure EBA of current treatment regimens for NTM-PD.

Three consecutively admitted patients were followed with weekly spot sputum cultures, which is part of the routine clinical care in the first phase of treatment. Patient 1 was a 68-year-old female with an unremarkable clinical history diagnosed with nodular-bronchiectatic Mycobacterium intracellulare pulmonary disease. Patient 2 was a 74-year-old female with a history of asthma and diagnosed with nodular-bronchiectatic Mycobacterium chelonae pulmonary disease. Patient 3 was a 65 -year-old female with a history of chronic obstructive pulmonary disease, now diagnosed with severe fibrocavitary $M$. avium pulmonary disease.

Alongside routine cultures, we performed quantitative cultures to assess the mycobacterial load in the sputum samples. The patients were followed the first month to assess the early bactericidal activity. In total, five sputum samples were collected from every patient. The first sample was collected before the start of treatment; thereafter, we collected a spot sputum sample each week for 1 month. The first (baseline) sputum was collected while patients were admitted; thereafter, patients collected sputum at home once a 